\title{
MARMOUSI-MODEL DATA SET: MACRO MODEL VERIFICATION AND PRESTACK DEPTH MIGRATION
}

\author{
Roland Marschall and Jens Thiessen \\ Prakla Seismos AG \\ Buchholzerstrasse 100 PO Box 510530 \\ 3000 Hannover 51 \\ Germany
}

\begin{abstract}
On January 19, 1990 we received the Marmousimodel data and the description of the data set (2-D seismic line, 240 shots with 96 channels each, wells 1504 and 9004). Then, on March 19, 1990 a new data set was received ( 5 shots have been corrected) plus an additional well 2700 including a VSP. The VSP, however, was never used by us. Our work was performed in two phases:

PHASE 1: conventional processing (including DMO) up to poststack time migration.

PHASE 2: Derivation of the macro-model and subsequently shotgather-oriented prestack depth migration.

In principle, two approaches exist to solve the problem caused by depth migration. The problem is that one has to specify the velocity as a function of coordinates $\mathrm{x}$ and $\mathrm{z}$ through which the observed wavefield is extrapolated using a rigorous version of the one way wave equation.

The two alternate approaches are either the FOCUSSING-APPROACH, (i.e. a guess of the macro model is refined by e.g. iterative shot migration) or the MACRO-MODELVERIFICATION-APPROACH (i.e. the macromodel is checked and corrected by wavefrontmodeling, based on at least two shot-geophone vectors per CMP and interface, the distance between neighbouring CMPs being equal to or less than half the cablelength).
\end{abstract}

After updating the macro model with this approach depth migration is done only once.

With this approach, after having migrated the individual shotgathers prior to stacking them, one may display the CRGs (common-reflection point gathers) as the final QC-tool.

\section{Introduction}

The complete description of the entire dataset in terms of seismic data acquisition as well as welldata is given by Bourgeois et al. (this volume). The ultimate deadline for us for the treatment of the data was May 30, 1990 (i.e. the date of the workshop).

All results presented in the following discussion were available at the workshop. In section 4 of this article we show, in addition to the above mentioned results, the effects of data decimation at post depth-migration stage proving that the original coverage of $48-$ fold, i.e. 240 shot records, could be reduced to a large extent without significant degradation of the resulting section.

This was done to demonstrate that for projects like this, where one usually would have many 2-D lines available, the complete processing effort would be carried out for one testline only. The resulting decimation test at the end clearly would define the possible amount of data decimation, reducing therefore the workload for the remaining lines by a considerable amount. 
Of course, the underlying macro-models must be of high quality, but this requirement can be fulfilled by the macro-model updating approach based on wavefront-modeling.

\section{Data treatment}

The actual processing work was divided into two phases (Marschall and Papaterpos, 1989):

\section{Phase 1:}

Phase 1 is the conventional processing flow up to poststack time migration (see Fig. 1).

The first step was to check the amplitude decay of the data. As a result no residual amplitude correction had to be applied, as can be seen from the example (shotpoint 5400, channels 1, 48 and 96) in Fig. 2.

The second step was the determination of the source wavelet, i.e. the watergun array signature (bandwidth 0-10/40-60 Hz). Two assumptions were used, namely that the signature is maximum delay (Evans et al., 1984, Marschall, 1988a) and that its period $\mathrm{T}$ is related to the notch-frequency $\mathrm{fn}$ in its amplitude spectrum by $\mathrm{fn}=3 /(2 \mathrm{~T})$ (Safar, 1976).

This resulted in $\mathrm{T}=56 \mathrm{msec}, \mathrm{fn}=27 \mathrm{~Hz}$ and confirmed the wavelet estimate $W o(1 / Z)$ which was derived as $W o(Z)$, i.e. in its time reversed form from the near trace section (time gate 0.3-2.4 sec) based on averaged autocorrelations.

The spiking operator is then simply given as the inverse operator to the given maximum delay wavelet, i.e. $1 /$ Wo $(1 / Z)$. After spiking an Ormsby bandpass filter (0-10/40-60 Hz) was applied.

Fig. 3 summarizes the above steps, i.e. we have from top to bottom: Estimated minimum delay wavelet, amplitude spectrum (with annotated notch position), time-reversed minimum delay wavelet, spiking filter operator and Ormsby filter operator.

The third step was velocity analysis before and after application of the dip moveout (DMO)process. Figs. 4a,b show an example at shotpoint 4000 , proving the well-known fact that after DMO the analysis of velocities is much easier.

After stacking the final step of phase 1 is the poststack time migration. The poststack FD-time migration was carried out with 90-percent velocities (the $90 \%$ figure was derived from a migration velocity test).

Therefore after phase 1 the resulting database for the subsequent phase 2 (= macro model and prestack depth migration) contains:

- a near trace section (Fig. 5)

- a near trace time migrated section (Fig. 6)

- a 48-fold stack (Fig. 7)

- a poststack time migration (Fig. 8), where the three synthetic seismograms are also shown

- constant-offset sections (COS)

- CMP gathers (prior to NMO)

- three wells $(1504,2700,9004)$ i.e. sonic and density logs

\section{Phase 2:}

Phase 2 consists of the construction of the macromodel (velocities and velocity interfaces), followed by prestack depth migration of shotgathers (Marschall 1988b, Marschall and Papaterpos 1989, Zuurbier and Marschall 1989).

The first step was to select the actual number of velocity-interfaces to be interpreted in the poststack time migration. Six interfaces with numbers $10,30,35,40,50$ and 60 have been interpreted and were image-ray migrated, where for the image-ray migration the "first guess" of the actual velocity depth field; derived from the existing well data, was used (see Figure 9).

Interfaces 40 and 50 on the resulting depth section (left hand side) then had to be corrected based on the interpretation of the unmigrated near-trace section, since at least for these interfaces the stack does not represent a "zero-offset section".

These interfaces therefore have been computed by ray migration. The velocity field for interfaces 10 , 30,35 and 40 consists of individual velocity depth functions of the form $V(Z)=V o+a Z$, whereas for the deeper interfaces constant interval velocities have been applied.

From interface 50 downwards a constant interval velocity of $4400 \mathrm{~m} / \mathrm{sec}$ was used. This in fact shows our philosophy in solving the given problem: The depth section from mean sea level to interface 50 should be analyzed primarily by conventional poststack time migration, whereas the section below interface 50 is to be resolved by prestack depth migration. 
The interface numbers 10 to 60 are annotated in Fig. 8 as well. The resulting preliminary depth section (= macro-model) is shown in Fig. 9.

The second step involved checking the resulting macro-model with wavefront-modeling. Two offsets with the corresponding traveltimes per selected CMP and interface were used. The CMPs used are located in the western and eastern part of the given line.

The usual method of verifying the macro-model is to apply this checking procedure to the chosen interfaces over the entire line. However, this method was not tried here because of the complicated tectonic style in the central part of the section.

An example of this checking procedure is shown in Fig. 10.

In the upper part of Fig. 10 the basic principle of the velocity checking approach is explained: here a zero-offset ray and an offset ray (offset $x$ ) are used. The corresponding traveltimes $T_{0}$ and $T_{x}$ both refer to the same interface.

The depth difference $\Delta Z$ (=error function) shown indicates that

$$
v T_{\circ} / 2<\left[\left(v T_{x} / 2\right)^{2}-(x / 2)^{2}\right]^{1 / 2}
$$

which means that the velocity $v$ is too high. The error function then is transformed into a depthand space-variant velocity correction $\Delta v(x, z)$, giving

$(v+\Delta v) T_{o} / 2=\left\{\left[(v+\Delta v) T_{x} / 2\right]^{2}-(x / 2)^{2}\right\}^{1 / 2}$

or $\Delta \mathrm{Z}=0$

i.e. the correct depth of the interface under consideration. Further detail may be found in Marschall and Papaterpos (1989). An actual example for the Marmousi-line is shown in Fig. 10, lower part. Here two offsets, i.e. $200 \mathrm{~m}$ and 1000 $\mathrm{m}$, are used with the corresponding traveltimes. Part A refers to the first verification step, i.e. we use the velocity field $v(x, z)$ and calculate the corresponding three pairs of pseudo ellipses (=loci of constant traveltime) for the offsets $200 \mathrm{~m}$ and $1000 \mathrm{~m}$ respectively.

The offset/traveltime pairs used are annotated above the shotpoints 7500,8000 and 8250 . Note that in Figure 10 we show the resulting three pseudo ellipses for inline offset $200 \mathrm{~m}$ plus the resulting envelope (=tangent curve to these pseudo ellipses), whereas we only show the resulting envelope for offset $1000 \mathrm{~m}$ and not the corresponding three ellipses for this offset.

The velocity field $v(x, z)$ results in a depth difference of $Z=17 \mathrm{~m}$ at shotpoint 8000 . After updating the velocity field by $v(x, z)$ the calculation is repeated and the result is shown at the bottom. Clearly there is no depth difference between the two envelopes anymore, proving that the macromodel at this position for this particular interface is data consistent (since we only used measured quantities, i.e. offsets and traveltimes).

The resulting error function obtained by the velocity checking procedure showed that the initial interpretation of interface 35 had to be corrected, of course also influencing the interval between interface 35 and 40.

The updated version of the macro-model along with the new velocity field $v(x, z)$ is shown in Fig. 11. Note that, for example, interface 10 is now defined at both sides of the line in the updated version, and that interface 35 has disappeared.

This change was confirmed by the density information in the two wells 1504 and 9004 respectively as indicated in Fig. 12. Also the waterlayer was incorporated into the final model (Fig. 11 , interface 5).

In Fig. 13 we show the final interpretation of the poststack time-migrated section.

Interfaces correspond to the depth section (Fig. 11) as follows: $5,1=10,2=40,3=50,4=60$. Note that layer 5 is a waterlayer of constant thickness, which of course does not result from interpreting a seismic section, but is a predefined quantity.

Afterwards we calculated the corresponding zerooffset section from the final macro-model (=depth section). This digitized time section is shown in Fig. 14. The final velocity distribution applies to the whole section below and is given in Table 1.

Note that we also reduced the velocity below interface 50 from $4400 \mathrm{~m} / \mathrm{s}$ to $4200 \mathrm{~m} / \mathrm{s}$, since we were able to use two checkpoints at both sides, corresponding to the two segments of interface 60 shown in Fig.14. 


\begin{tabular}{|l|l|}
\hline INTERVAL & VELOCITY v(z) \\
\hline MSL to 5 & $1500 \mathrm{~m} / \mathrm{s}$ \\
5 to 10 & $1605 \mathrm{~m} / \mathrm{s}+0.265 \mathrm{z}$ \\
10 to 40 & $1760 \mathrm{~m} / \mathrm{s}+0.650 \mathrm{z}$ \\
40 to 50 & $2800 \mathrm{~m} / \mathrm{s}$ \\
\hline below 50 & $4200 \mathrm{~m} / \mathrm{s}$ \\
\hline
\end{tabular}

Table 1 Final velocity field

Fig. 15 shows an overlay of the zero-offset section derived from the final macro-model including diffractions and the unmigrated near-trace section, giving an additional check. After updating the velocity field on a layer-by-layer basis as discussed above, the final macro-model was obtained. A coloured display of the final macro model is presented in colour figure 7.

The third step i.e. prestack depth migration was carried out next. Here each shotgather is depth-migrated individually including zero trace padding. One-sided padding with 120 traces was applied as suggested by the final macromodel. Prior to the stacking of the individually migrated shotgathers a muting scheme has to be derived and the so-called CRG-gathers (commonreflection point) have to be displayed.

The CRGs give the final quality control tool, because each event must be horizontally aligned in these gathers. If there is residual move-out then this means that the underlying macro-model must be corrected in terms of velocities and/or velocity interfaces. The final depth-migrated section is obtained by stacking the individual shotgathers (Fig. 16). For this result no mute has been applied at all.

In addition Fig. 17 gives the result after having applied what we thought was the optimum muting scheme. The mute parameters can be deduced from the left hand side and right hand side of the resulting section (Fig. 17).

A useful modification here would be instead of padding zero traces to add REAL DATATRACES by applying the RECIPROCITY principle! This means that we would transform the given shotgathers into SPLIT-SPREAD-gathers prior to the depth migration process.

Interpretation (definition of macro-model):

Here usually the interpreter responsible for the actual area has to carry out the final interpretation.
As stated in the description of the model data set, presaliferous folded carbonate platform deposits are to be identified, in which a structural hydrocarbon trap is expected.

The resulting depth section now may be compared with the known solution, i.e. colour figure 1. Clearly the result shows that we have solved the target problem.

\section{Additional results}

As discussed, Fig. 16 gives the final result of prestack depth migration, where all individually depth-migrated shotgathers have been stacked without muting. Additional results here besides muting (see Fig. 17) are obtained by data decimation, i.e. we stack every n'th shot record only. The following table summarizes the results:

Fig. 18: stack of every 3rd shot record

Fig. 19: Stack of every 5th shot record

Fig. 20: Stack of every 7th shot record

Fig. 21: Stack of every 5 th record including muting

As can be seen, we have included one example, where a mute has also been applied to the individual shotgathers (Fig. 21). This result may be compared with Fig. 17. All other results are shown without any mute. Comparing the results (Figs. $16-21$ ) we concluded that even a decimation of the data set to $20 \%$ of the original volume gives an acceptable result in terms of the target-zone (i.e. below interface 50 of the macromodel).

\section{Summary}

Prestack shotgather depth migration based on a reliable macro-model has been applied to the dataset. 
The initial macro-model was checked and corrected by wavefront-modeling. The final depth migration was only carried out once.

A final decimation test showed that with respect to the target zone, i.e. below interface 50 of the macro model, only $20 \%$ of the data set still gives an acceptable result.

\section{References}

Bourgeois, A., Bourget, M., Lailly, P., Poulet, M., Ricarte, P., Versteeg, R. : Marmousi, the model and the data (this volume).

Evans, T., Schildt, P. and Marschall, R., 1984: A deconvolution method for implosive source type data: 54th SEG convention, Atlanta. Preprint, PRAKLA-SEISMOS AG, Hannover.

Marschall, R., 1988a: Power output and resolution (marine sources): Preprint, PRAKLASEISMOS AG, Hannover.
Marschall, R., 1988b: Saltdomes: Informatik Fachberichte 179, Simulationstechnik, 5. Symposium, Aachen, W. Ameling (Hrsg.) Springer Verlag, Heidelberg.

Marschall, R. and Papaterpos, M., 1989: Some aspects of prestack shotgather based depth migration with special emphasis on macro model verification: 1st Hellenic Geophysical Congress, Athens, April 19-21, 1989. Preprint, PRAKLASEISMOS AG, Hannover.

Safar, M.H., 1976: Calibration of marine seismic sources using a hydrophone of unknown sensitivity: Geophysical Prospecting, Vol. 24, 328-333

Zuurbier, N. and Marschall, R., 1989: Migration velocity by wavefront processing: 51st EAEG meeting, Berlin. Preprint, PRAKLA- SEISMOS AG, Hannover. 


\section{List of figure captions}

Fig. 1: Processing flowchart

Fig. 2: Amplitude decay curves

Fig. 3: Watergun wavelet

Fig. 4a: Velocity analysis at SP 4000 (prior to DMO)

Fig. 4b: Velocity analysis at SP 4000 (after DMO)

Fig. 5: Near-trace section

Fig. 6: Near-trace time-migrated section

Fig. 7: Final stack

Fig. 8: Time migration

Fig. 9: First macro-model

Fig. 10: Example for velocity checking

Fig. 11: Final macro-model

Fig. 12: First macro-model, correction

Fig. 13: New interpretation of time migration

Fig. 14: Zero-offset section (derived from final macro-model), without diffractions

Fig. 15: Overlay of near-trace section and zero offset section (derived from final macro-model), with diffractions

Fig. 16: Prestack depth migration of every shot, without muting

Fig. 17: Prestack depth migration of every shot, with muting

Fig. 18: Prestack depth migration of every 3rd shot, without muting

Fig. 19: Prestack depth migration of every 5th shot, without muting

Fig. 20: Prestack depth migration of every 7th shot, without muting

Fig. 21: Prestack depth migration of every 5th shot, with muting 


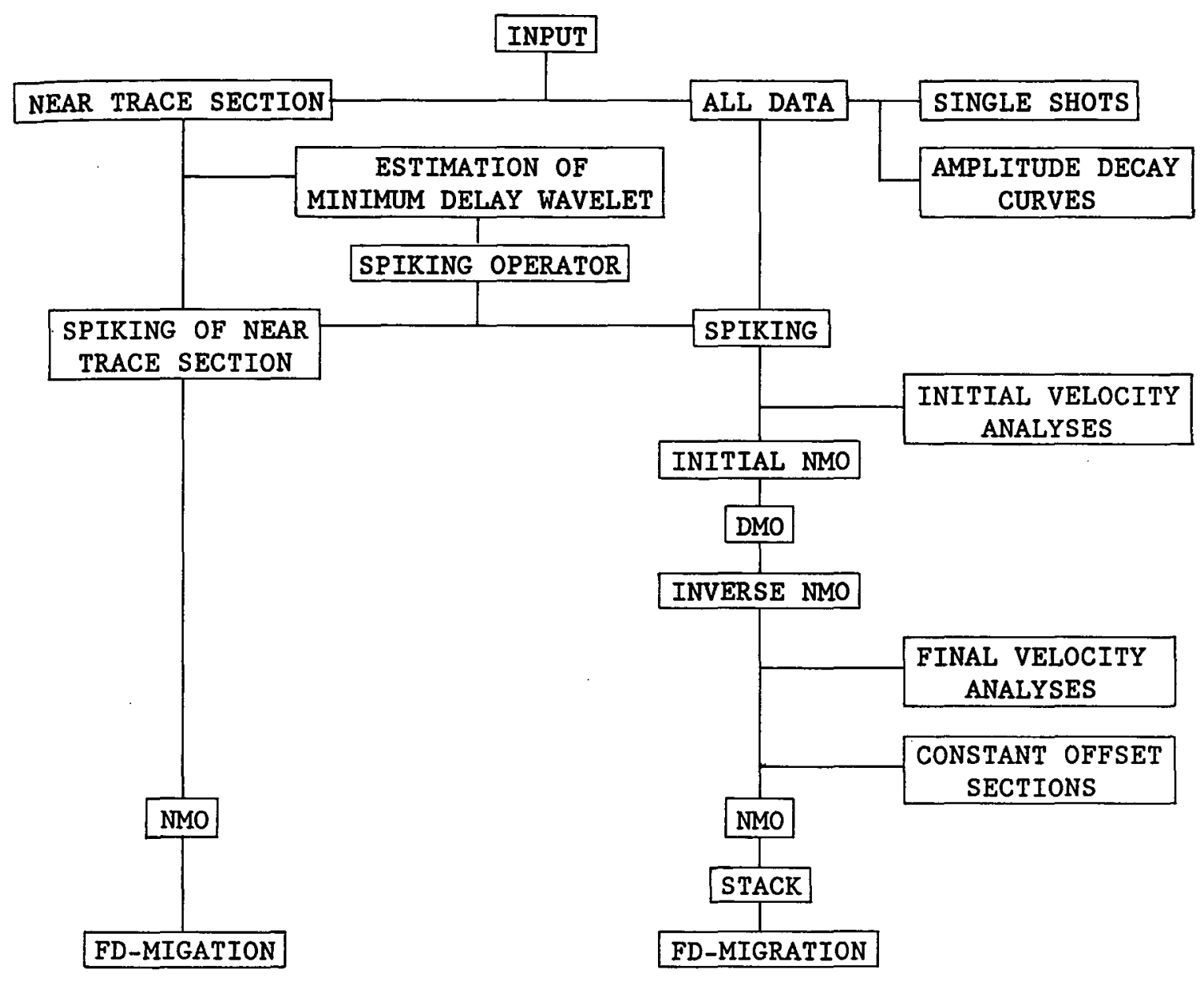

Fig. 1: Processing flowchart 

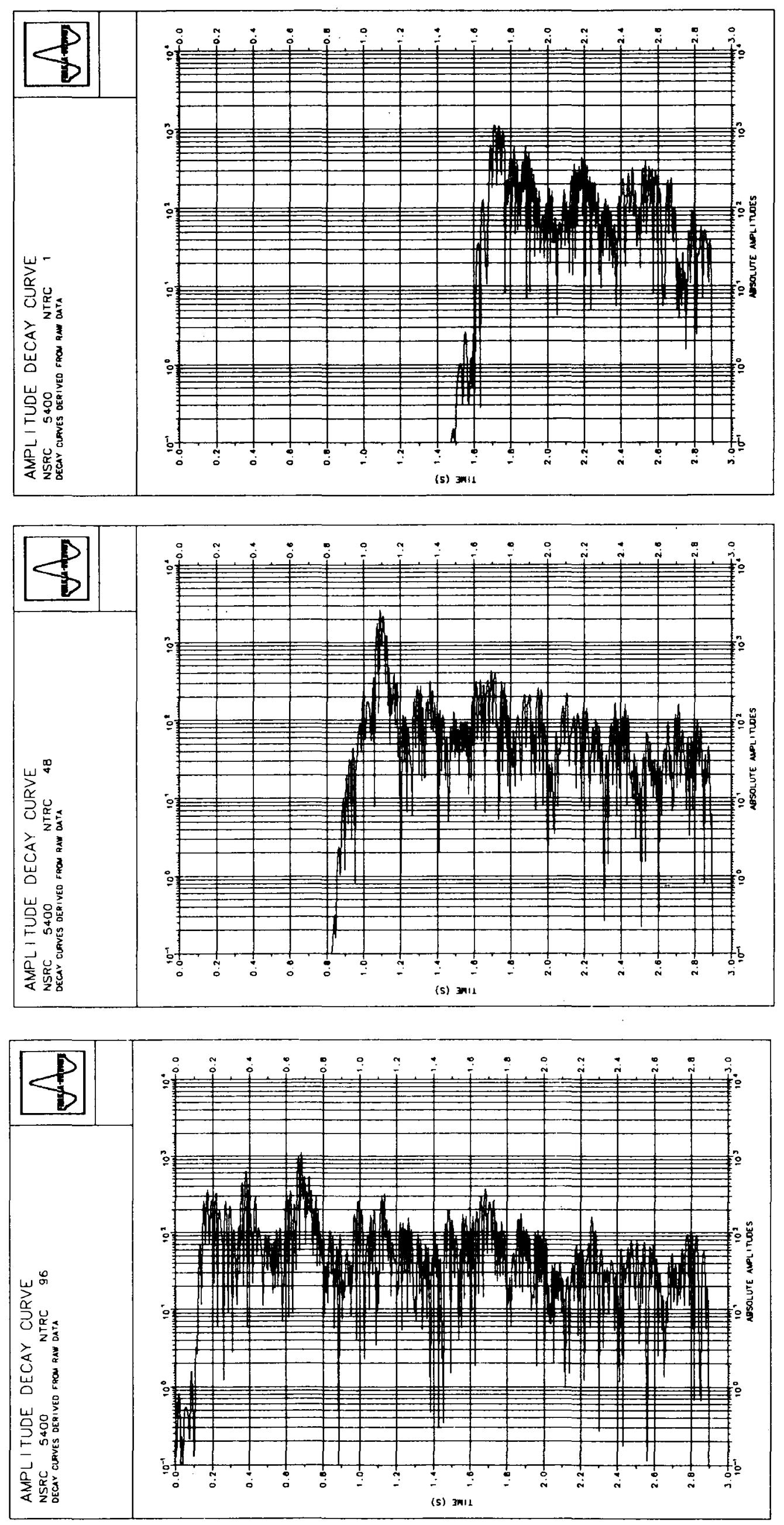

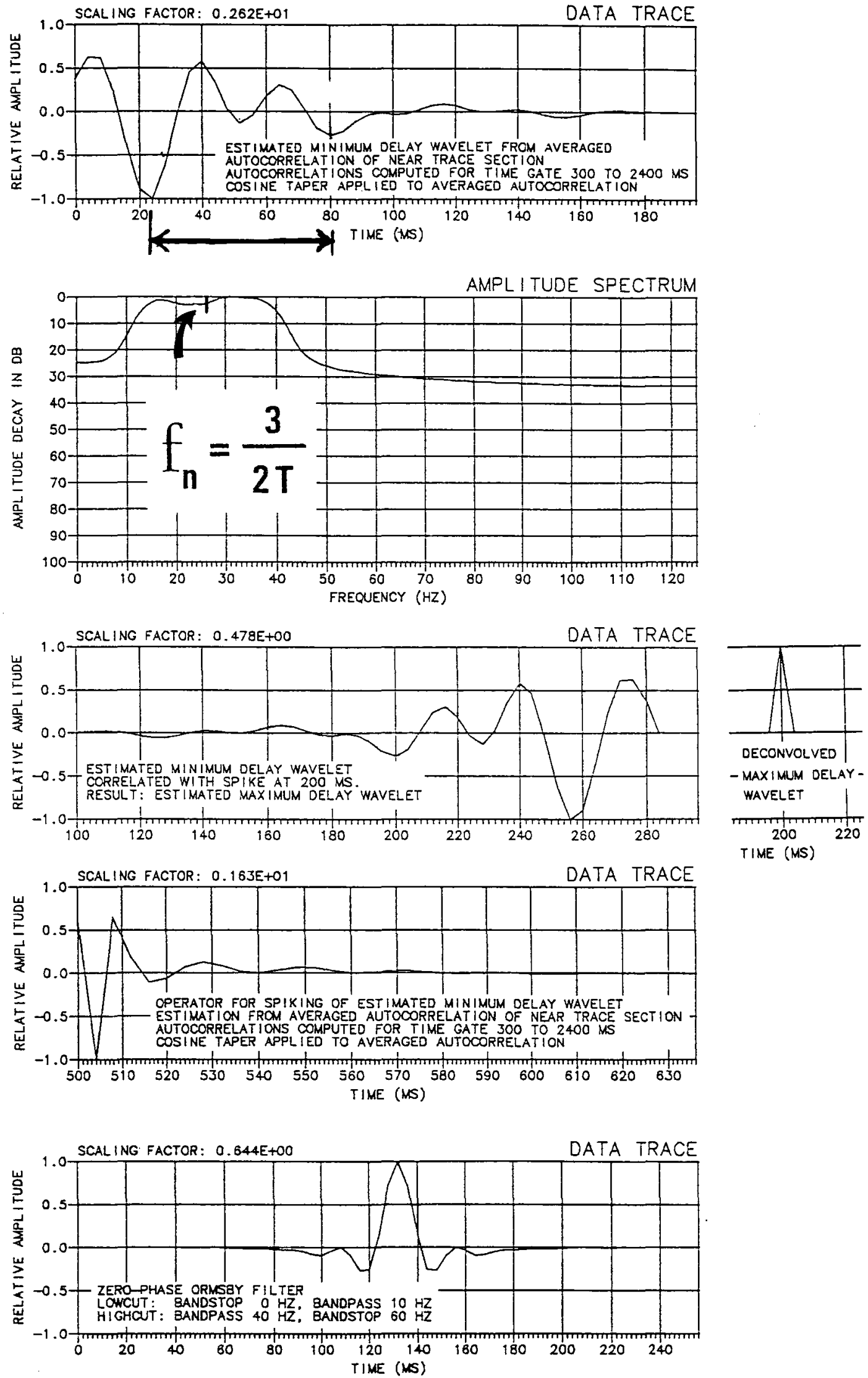

Fig. 3: Watergun wavelet 

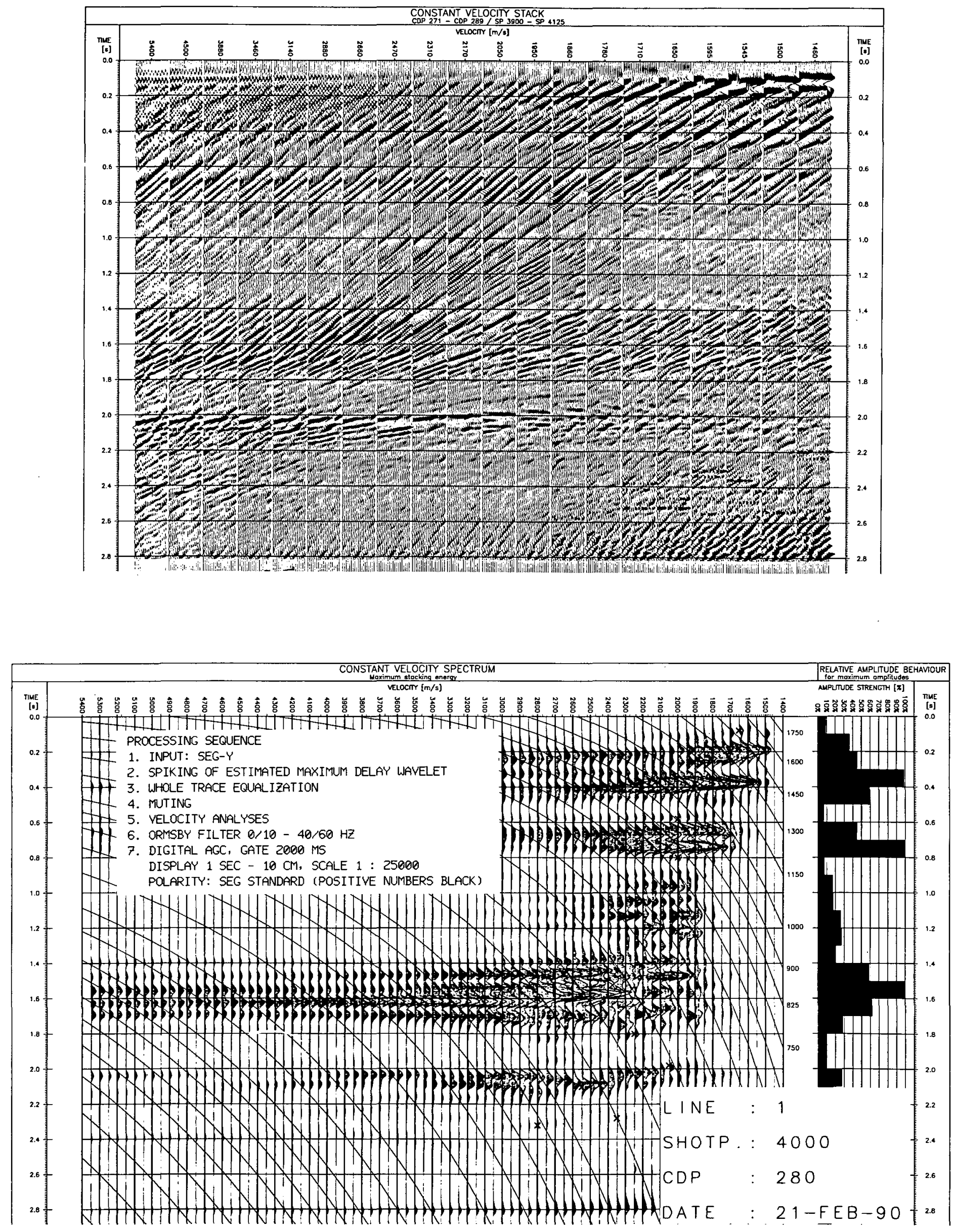

Fig. 4a: Velocity analysis at SP 4000 (prior to DMO) 

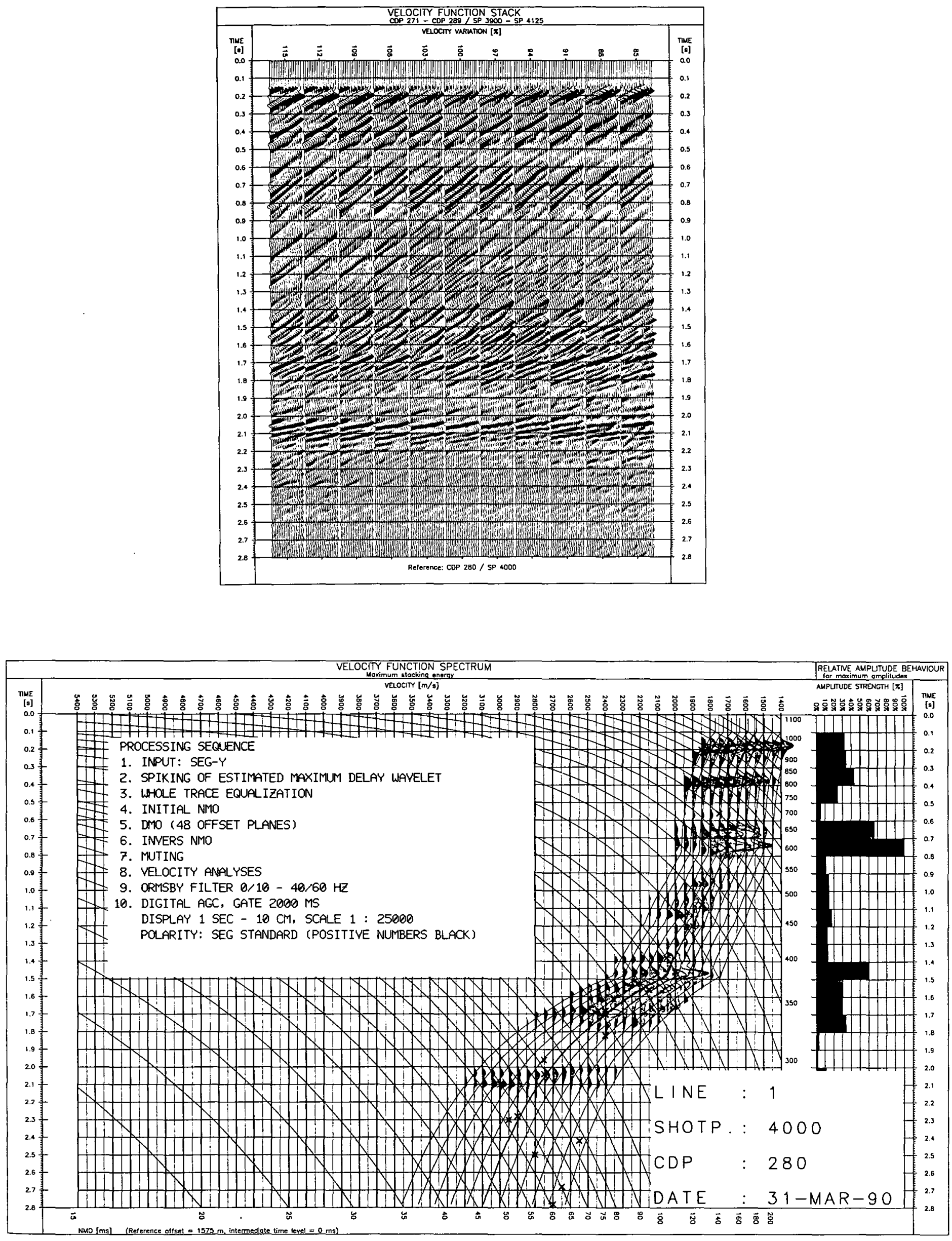

Fig. 4b: Velocity analysis at SP 4000 (after DMO) 


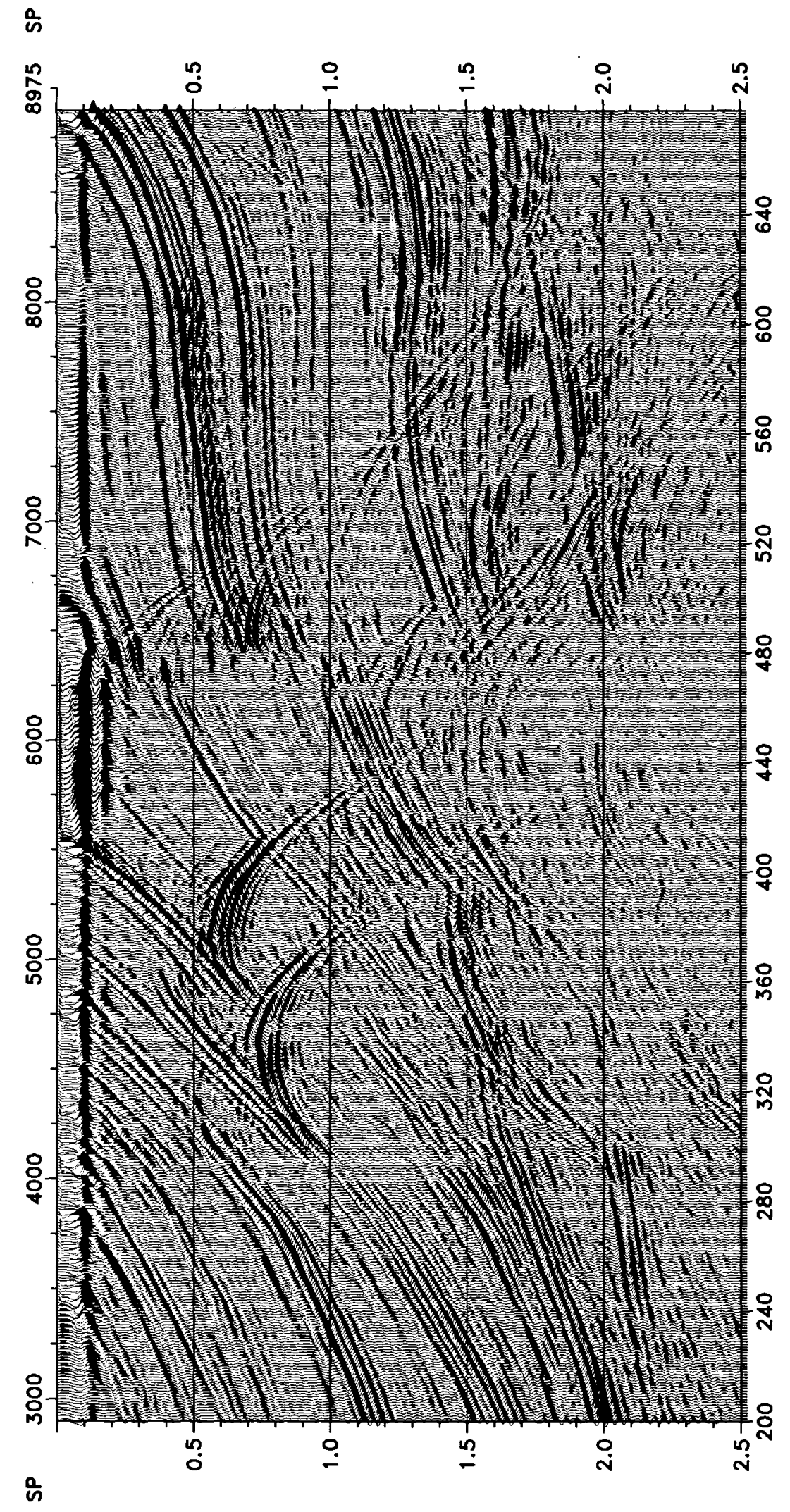

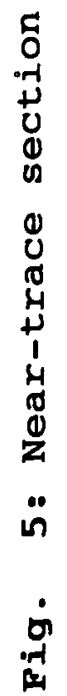




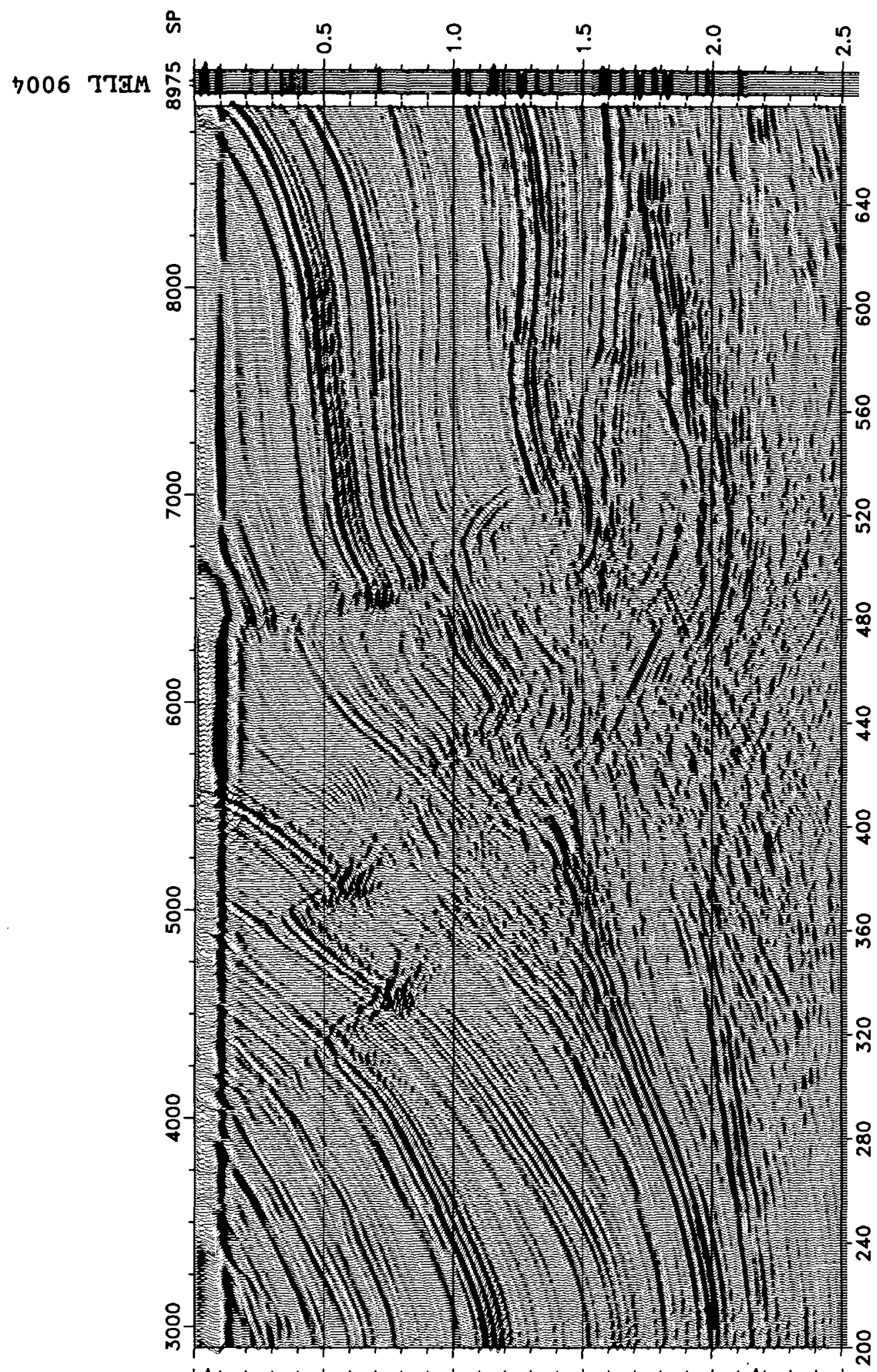

00L2 TTGM

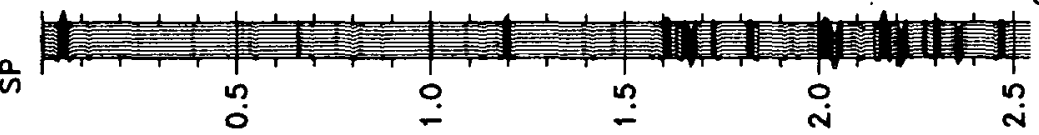

HOST TTAM

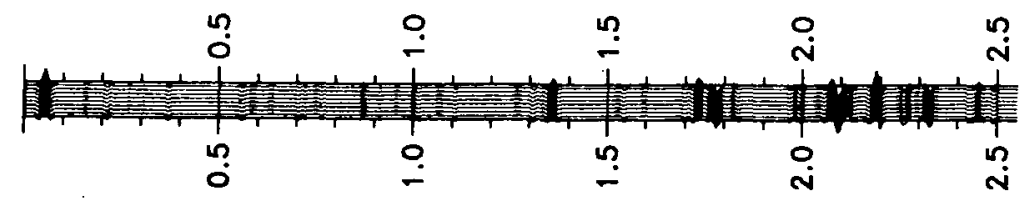




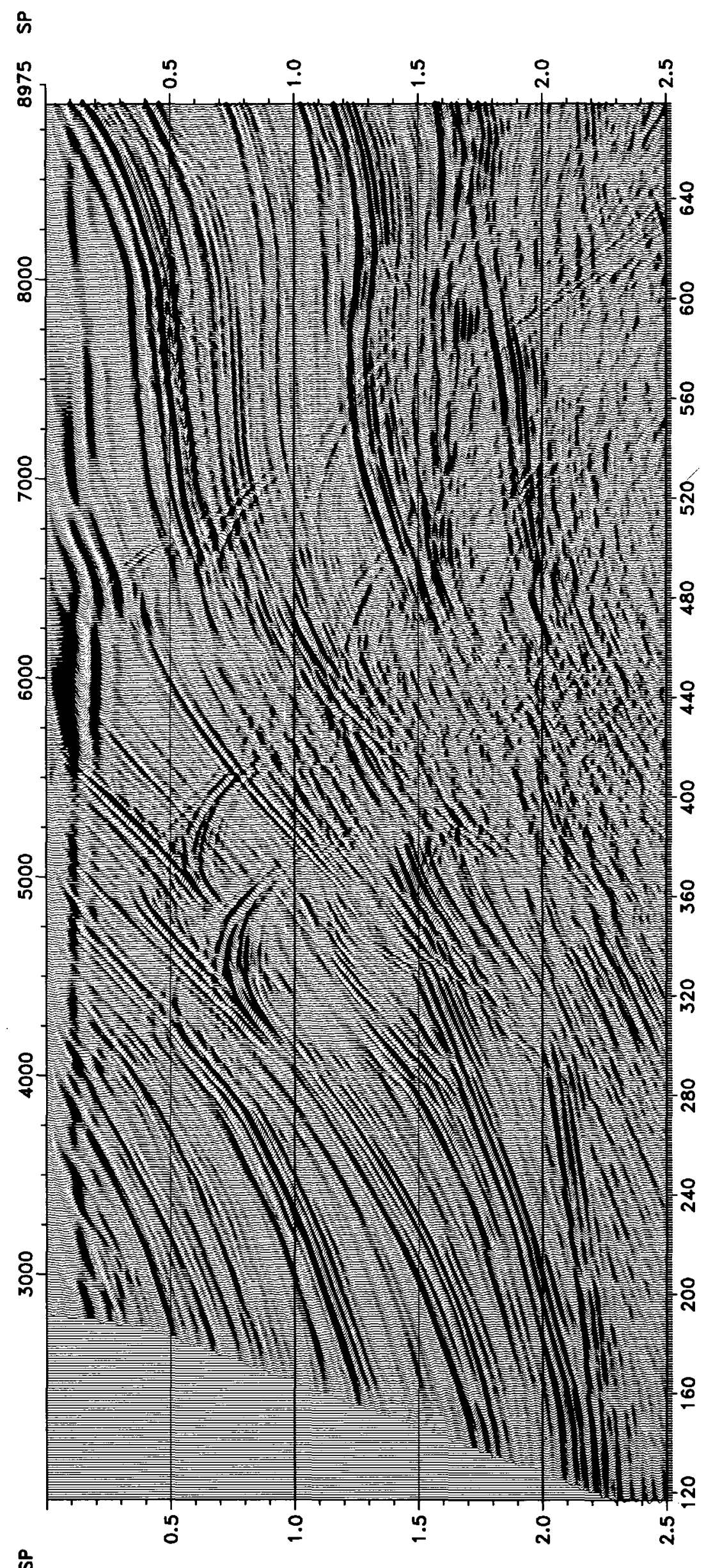

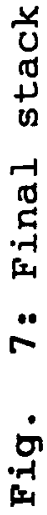




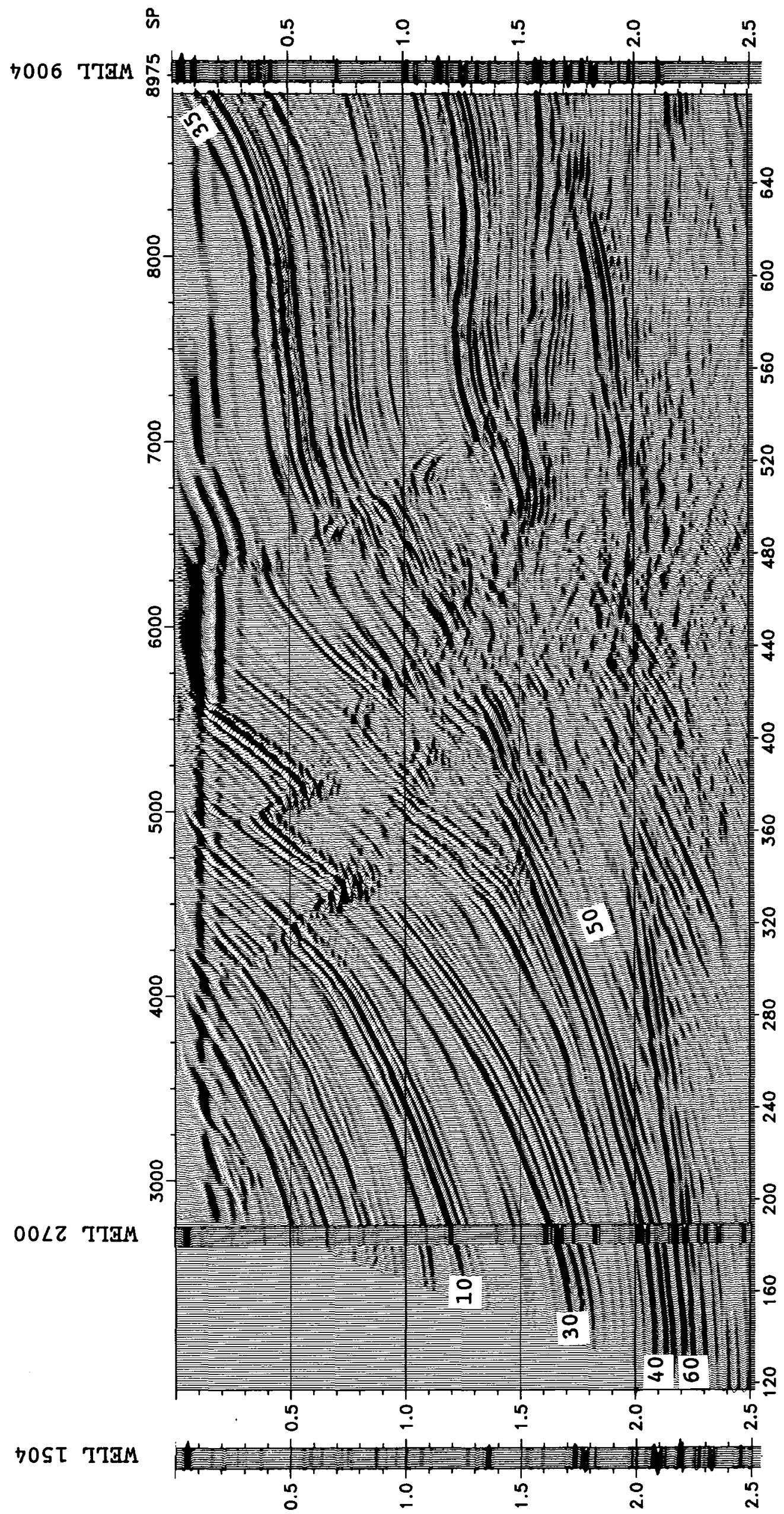

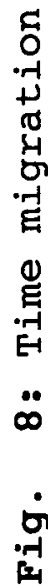




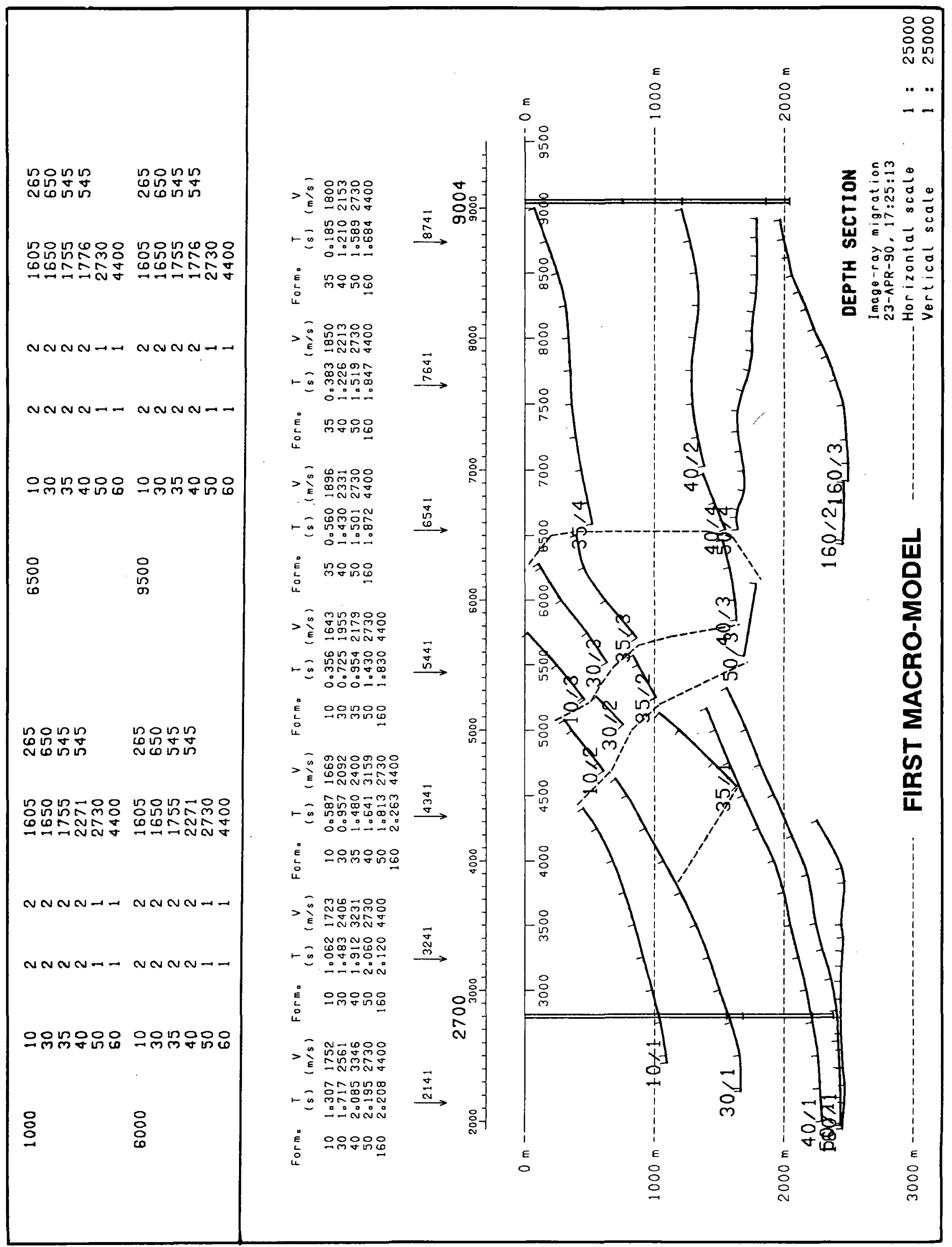

Fig. 9: First macro model 


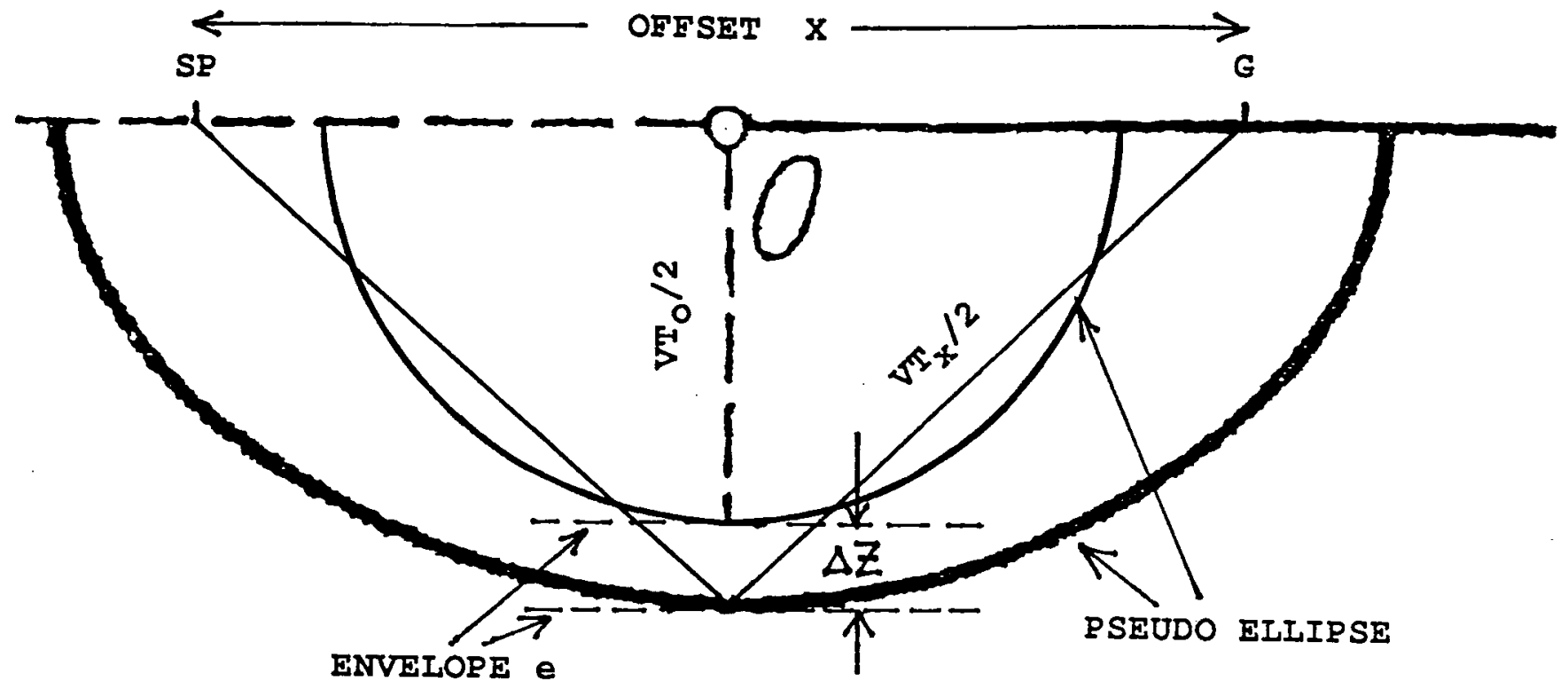

EXAMPIE
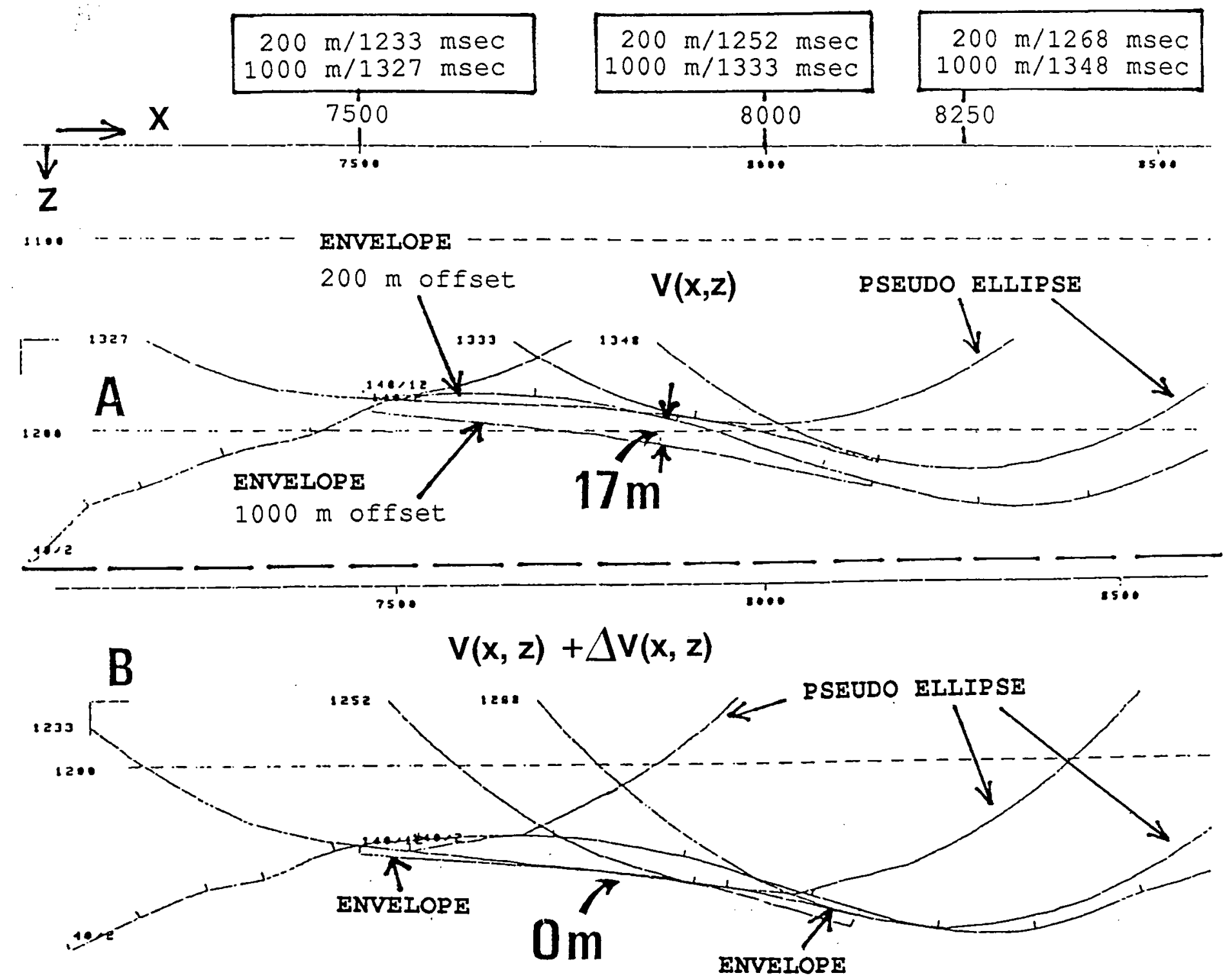

Fig. 10: Example for velocity checking 


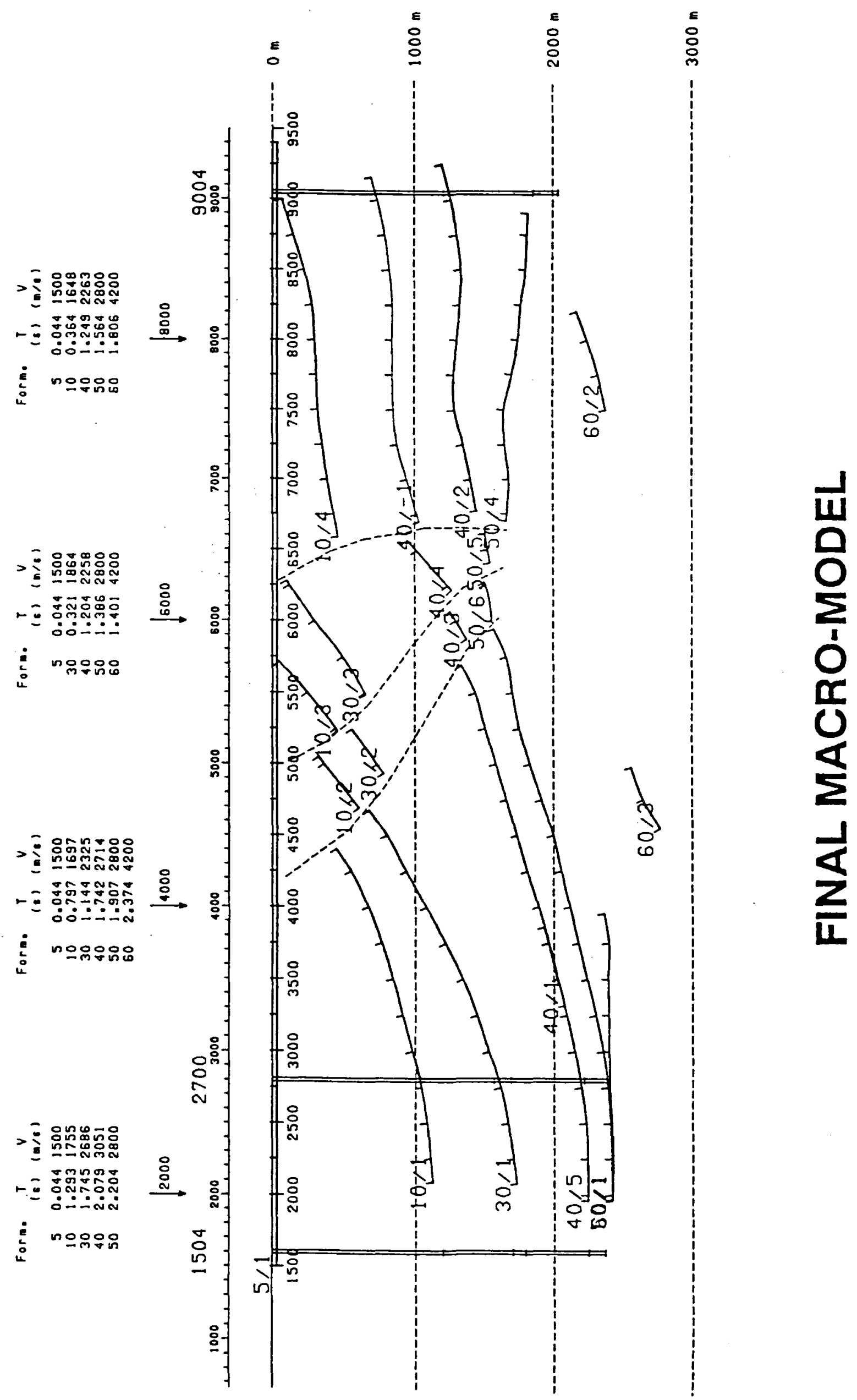

Fig. 11: Final macro-model 


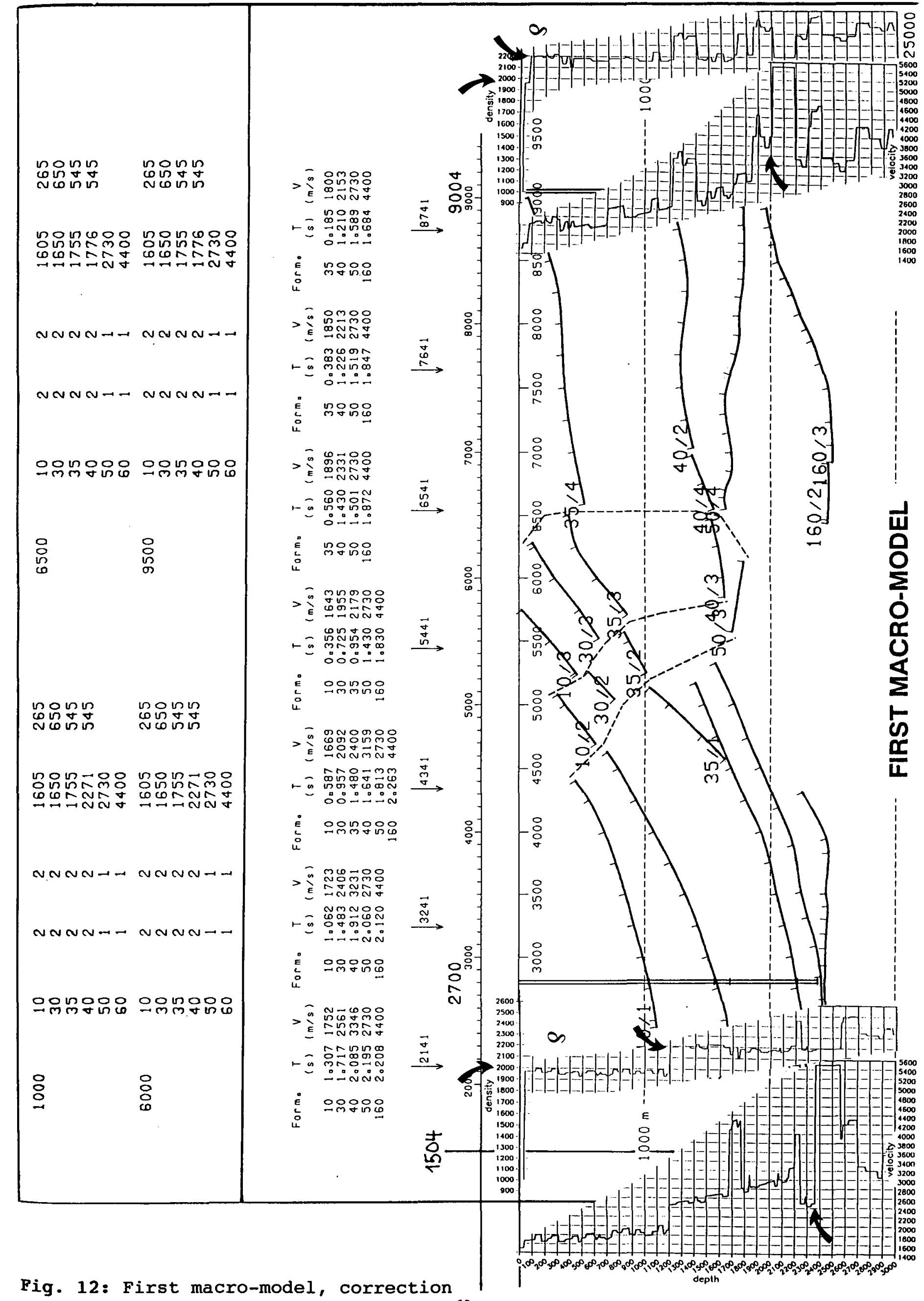




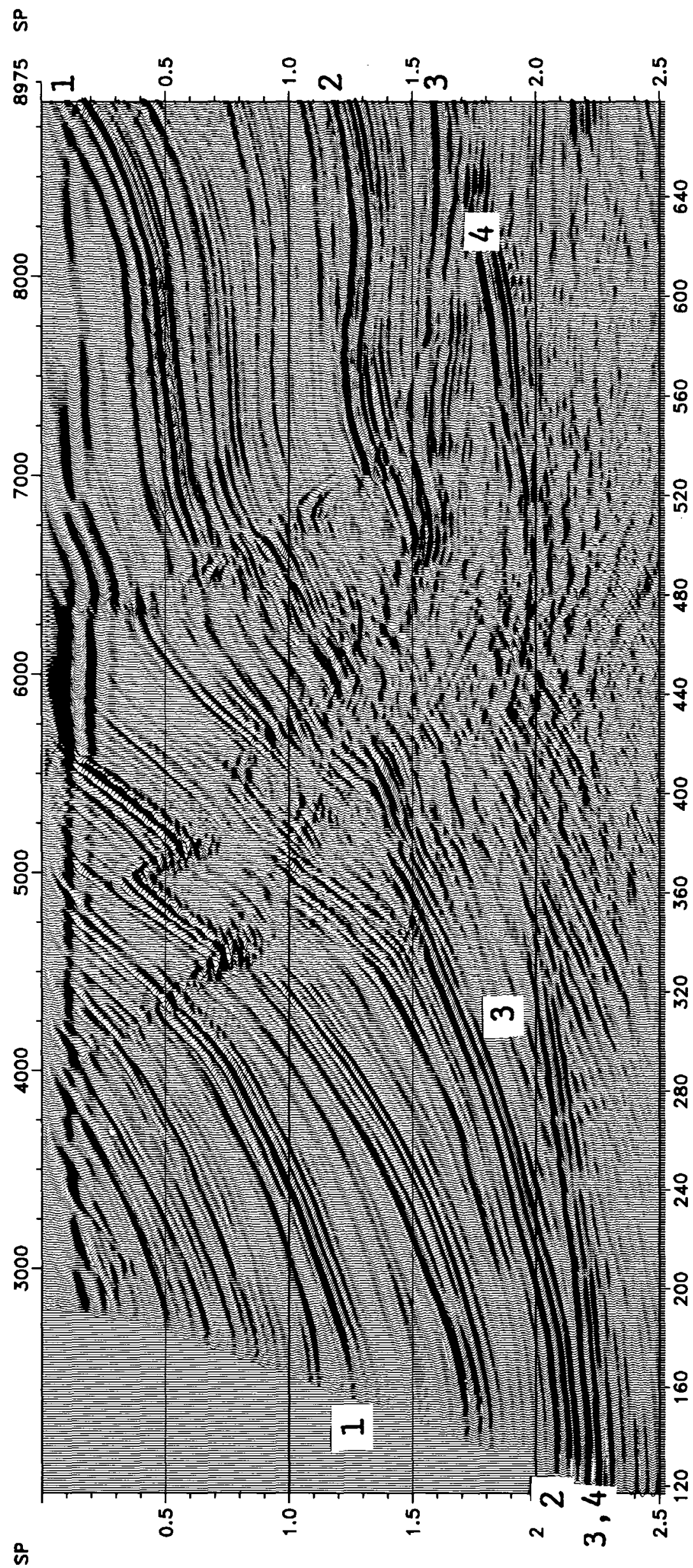

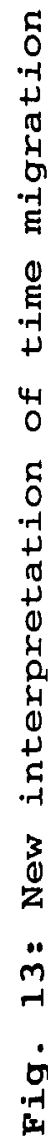




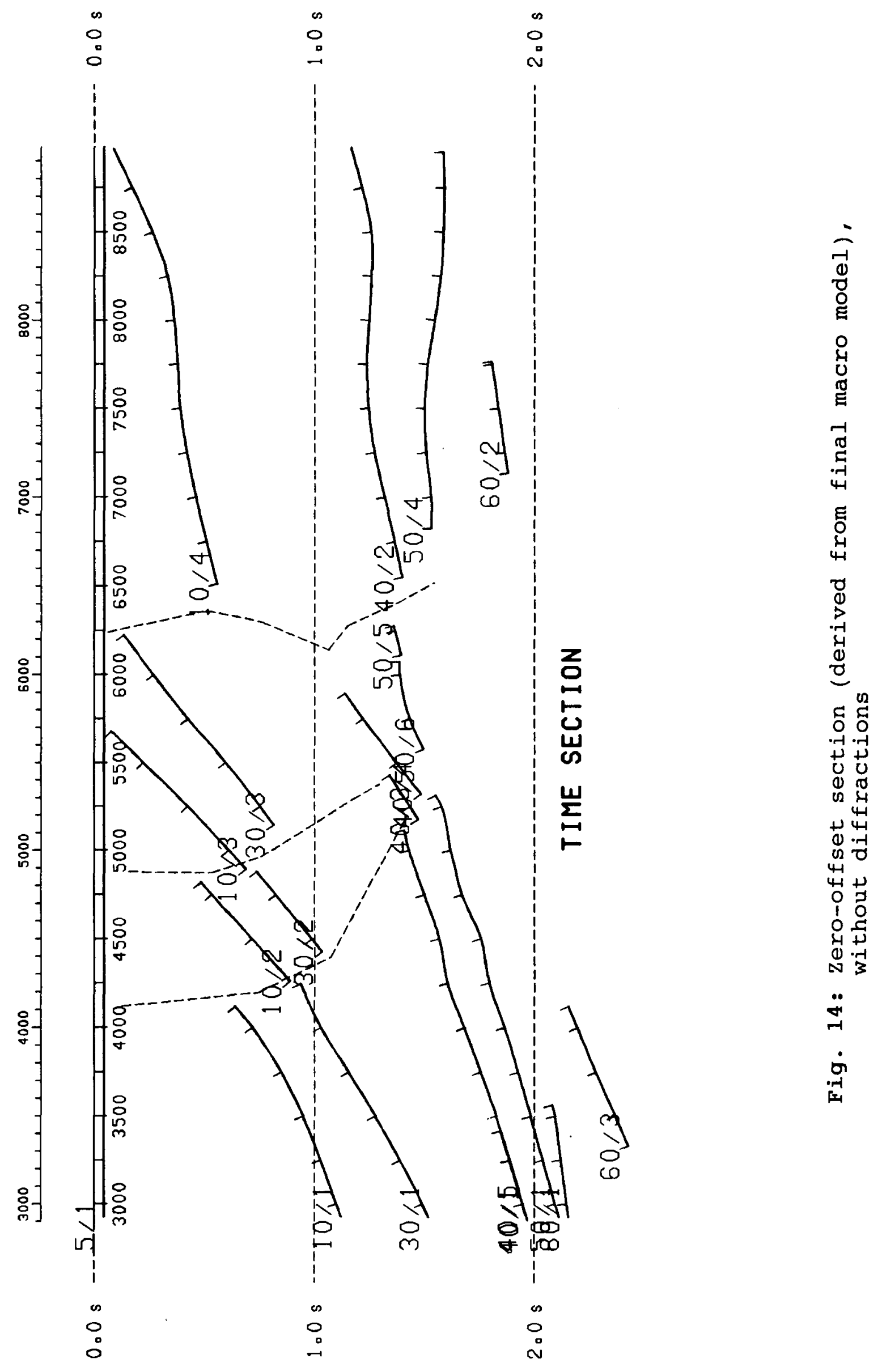




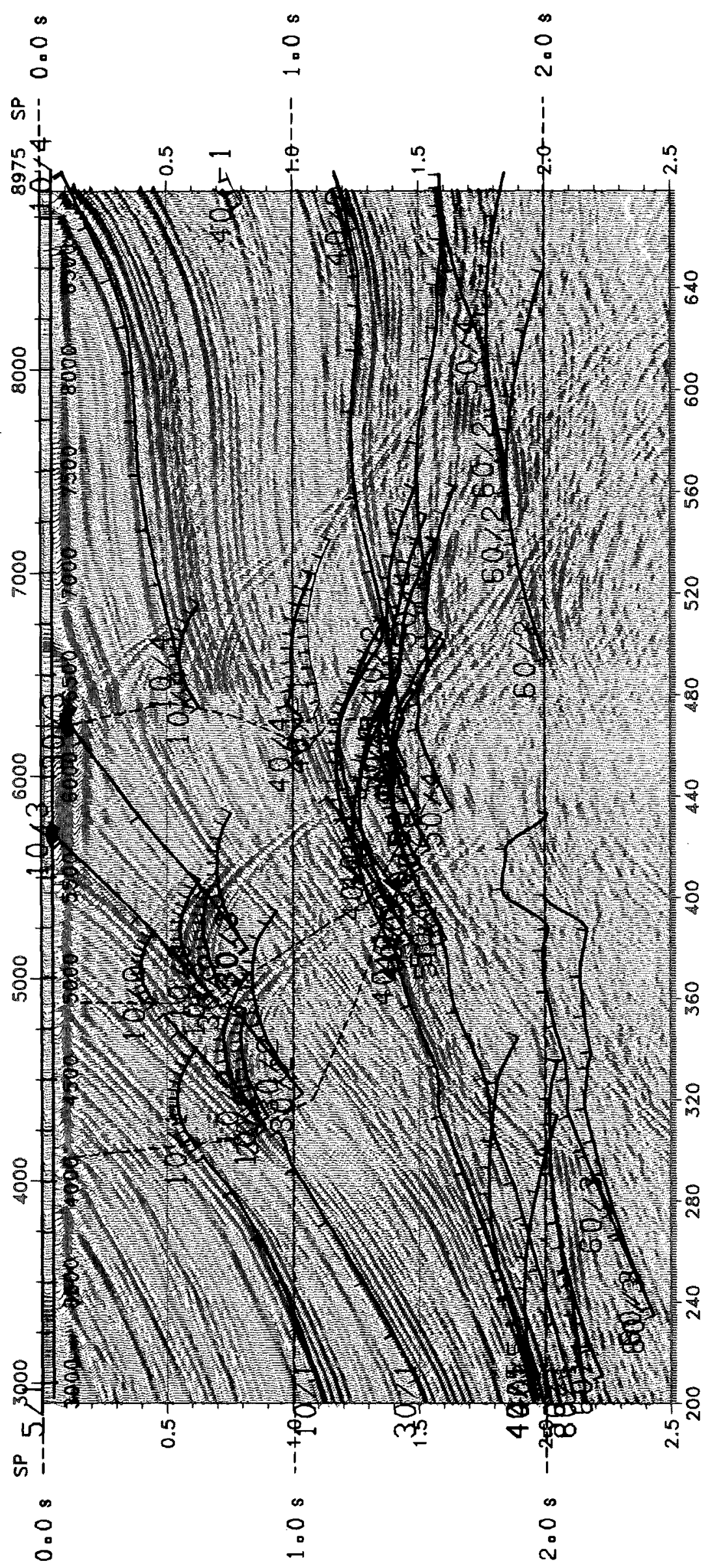

D5 


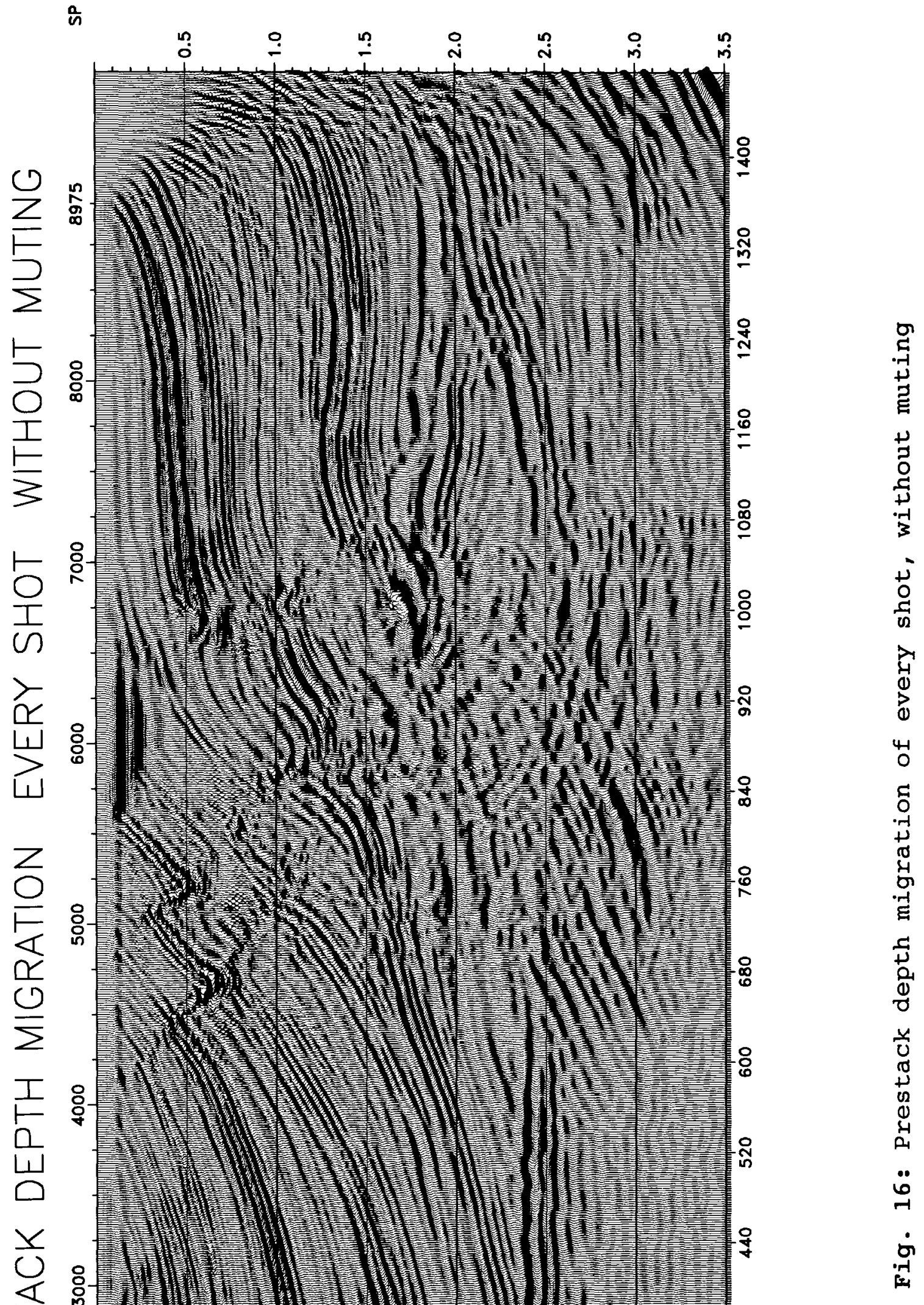




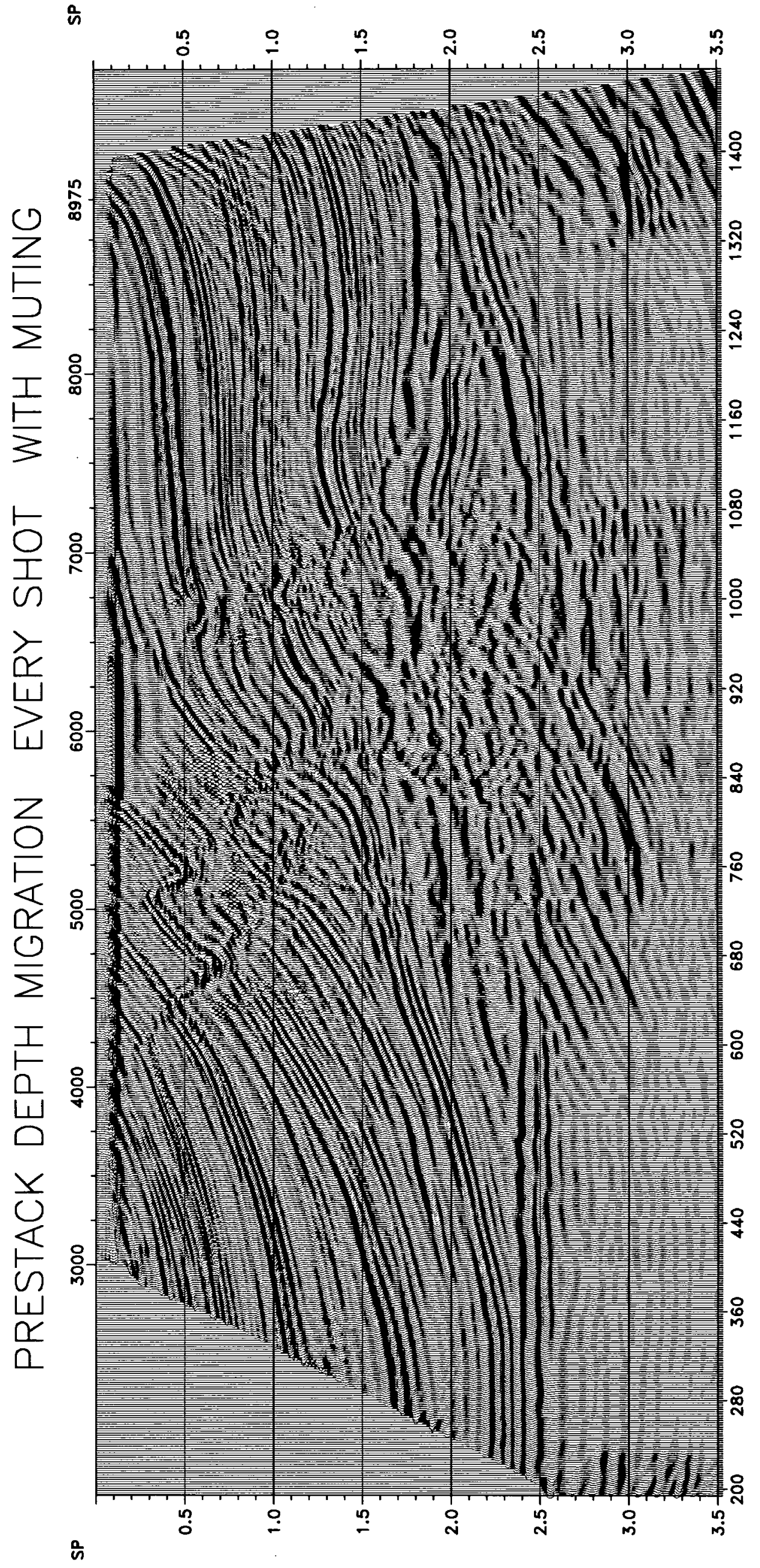

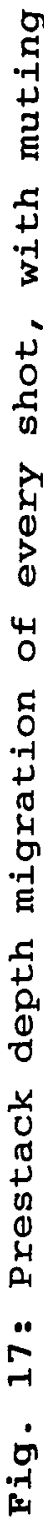




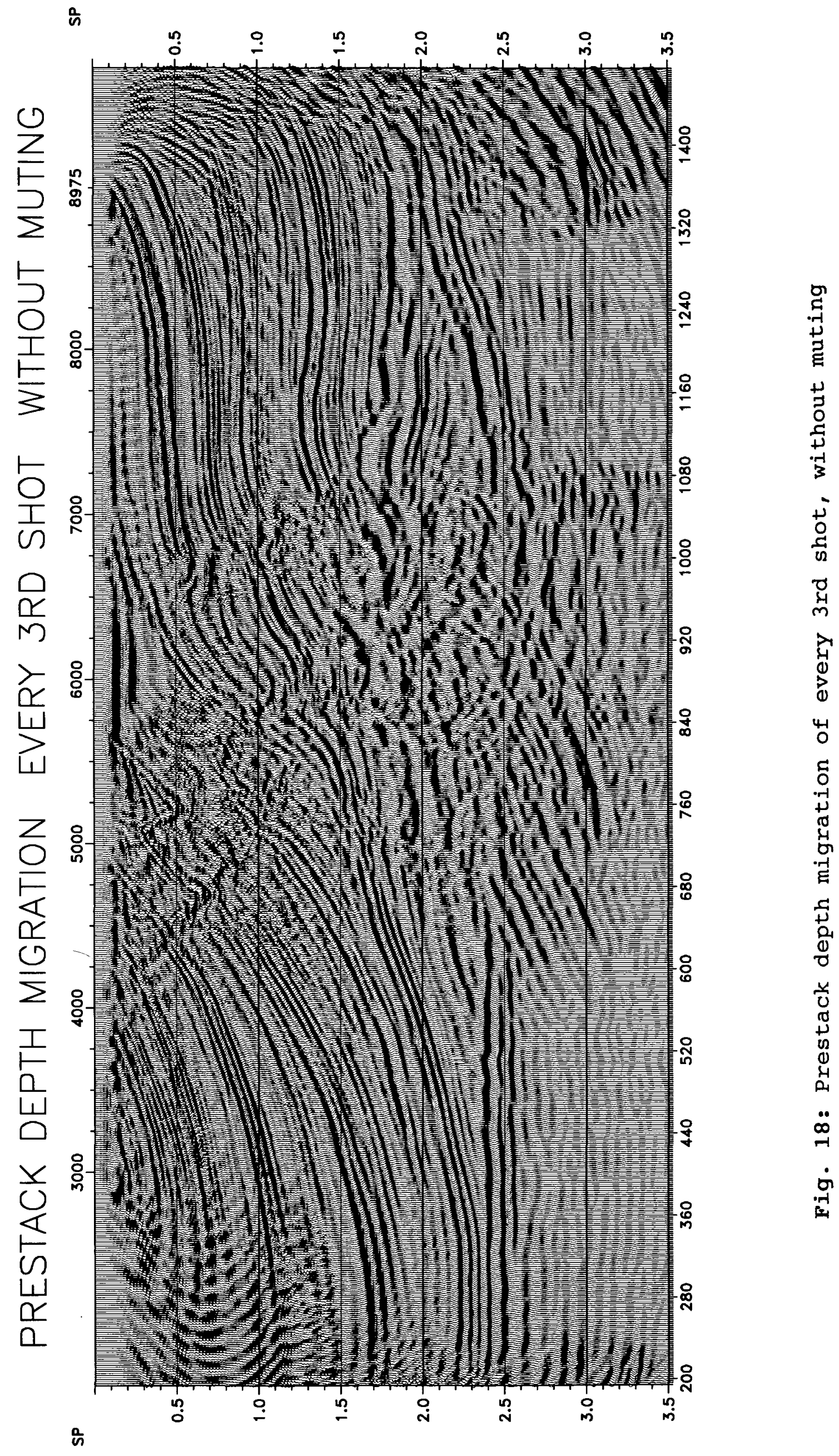




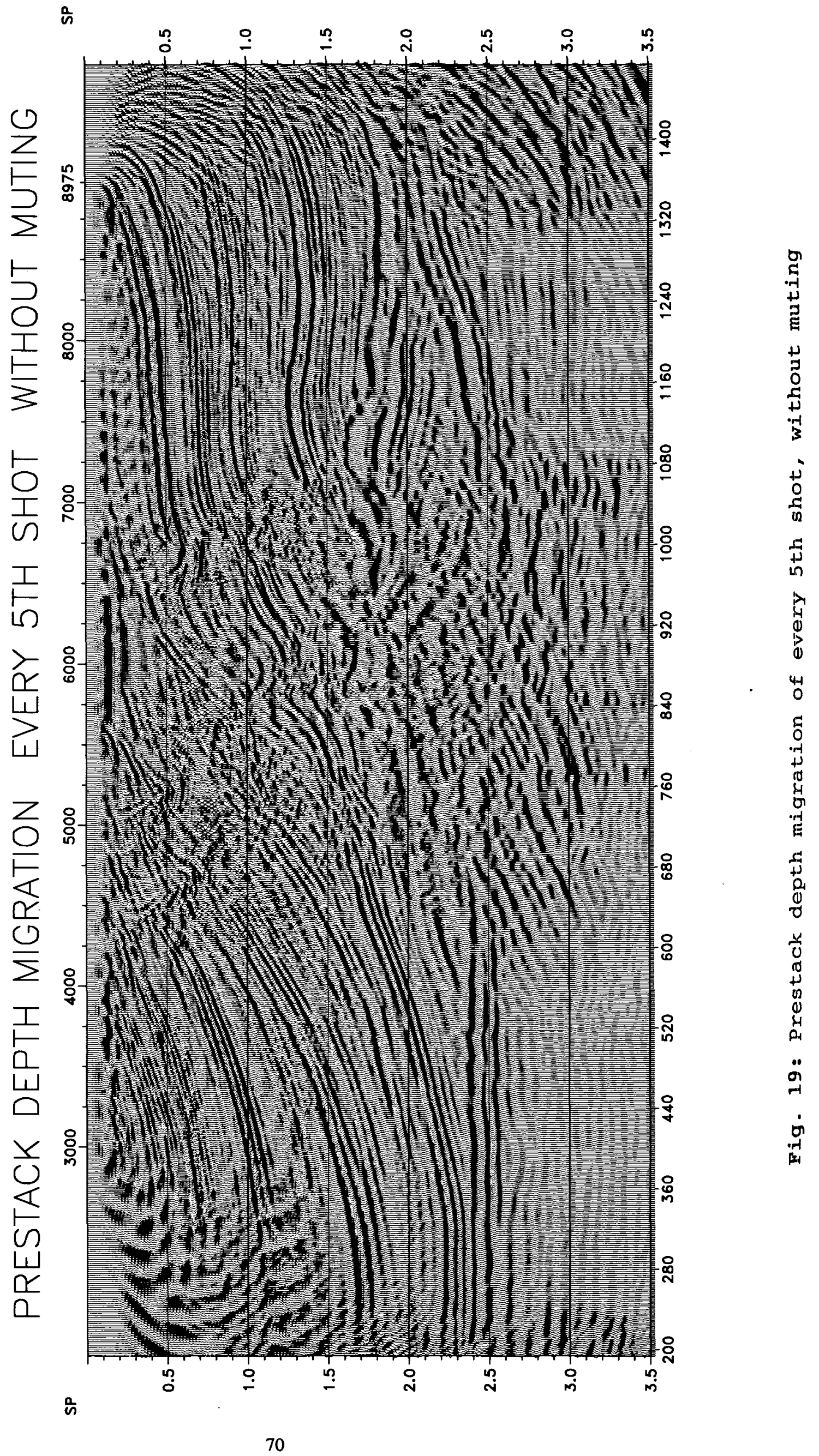




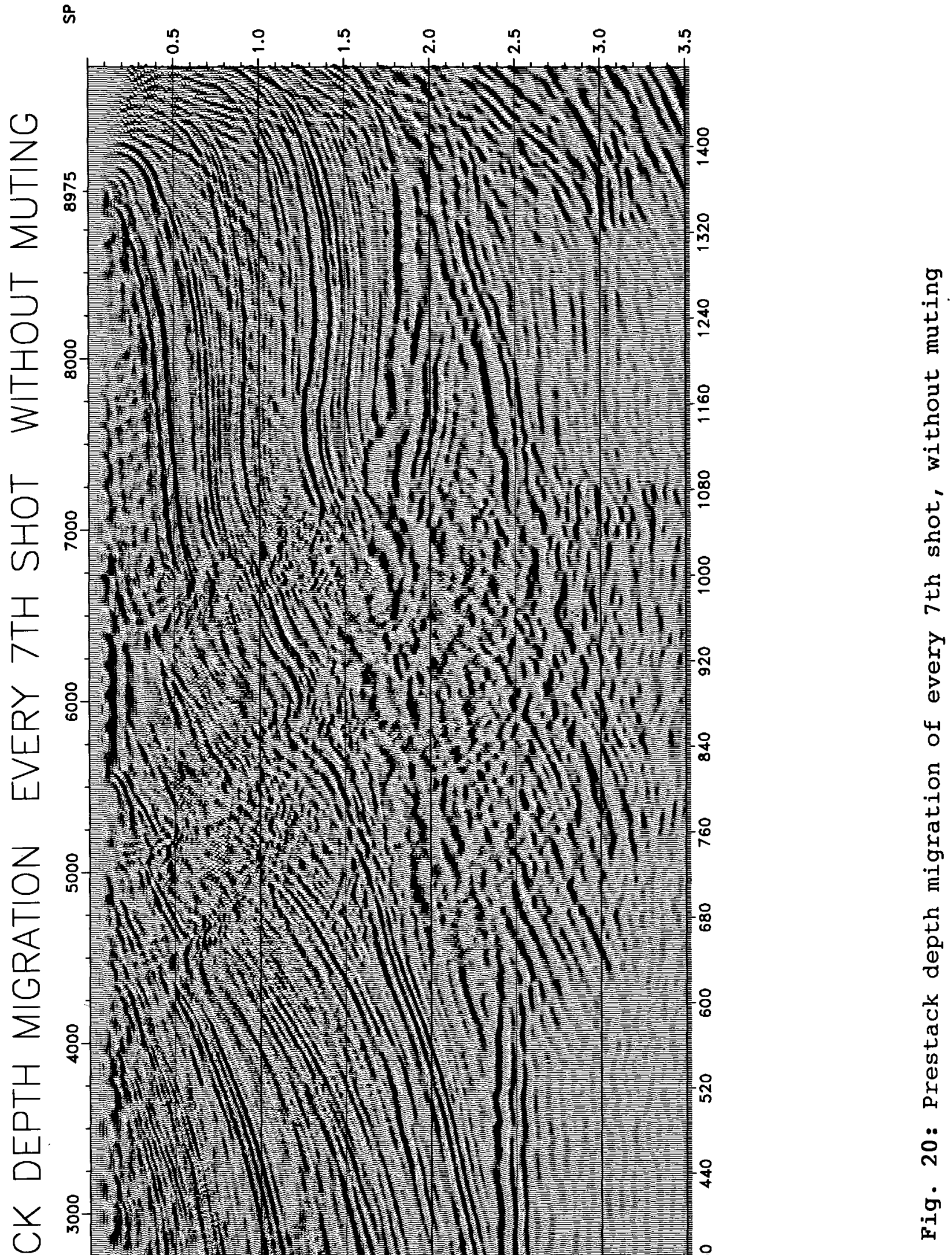




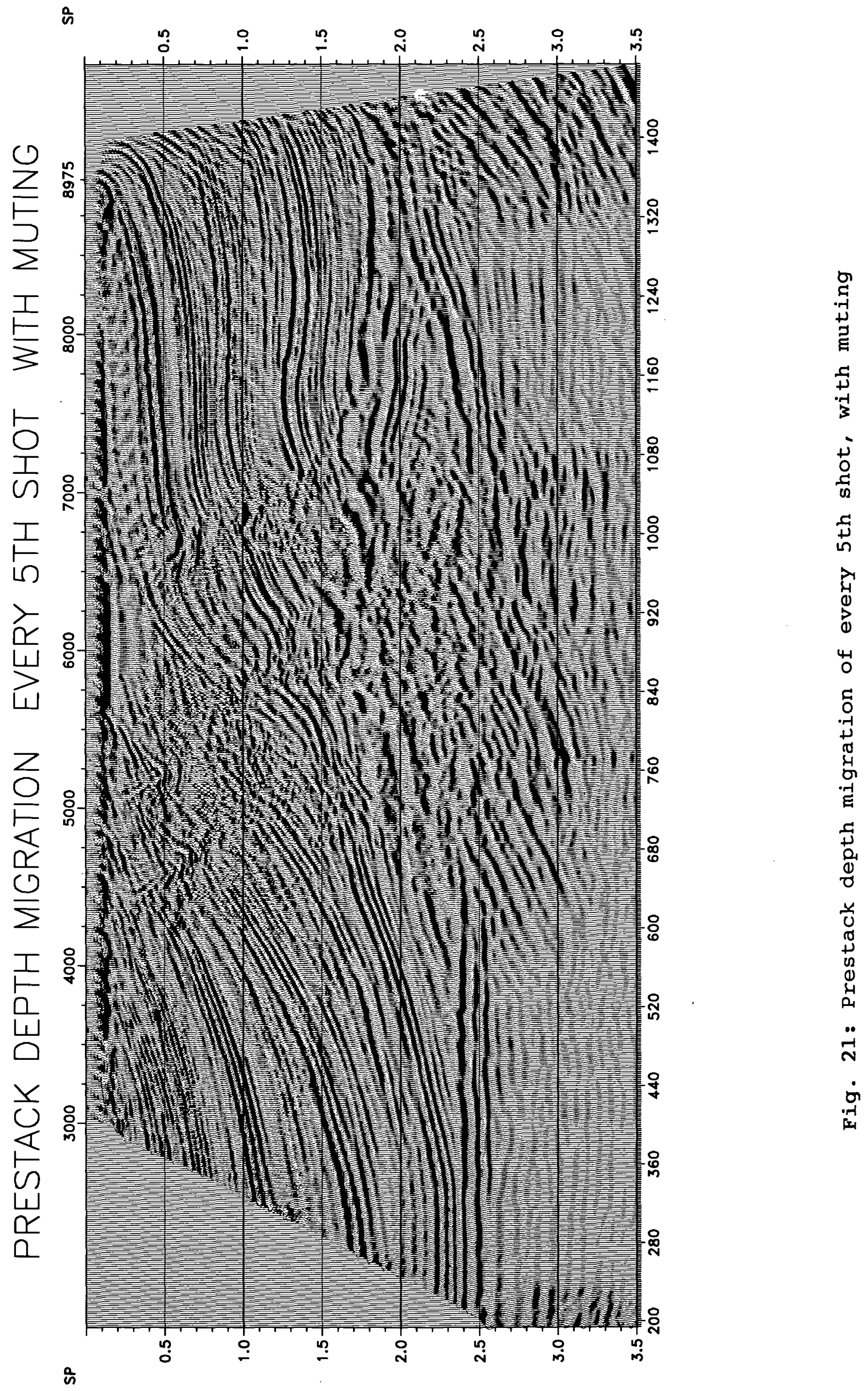

